\title{
Restoring Redundancy to the MAP Propulsion System
}

\author{
James R. O'Donnell, Jr., Ph.D., Flight Dynamics Analysis Branch \\ Gary T. Davis, Propulsion Branch \\ David K. Ward, Systems Engineering Branch \\ NASA Goddard Space Flight Center \\ Greenbelt, MD 20771 USA
}

\section{SUMMARY}

The Microwave Anisotropy Probe (MAP) is a follow-on to the Differential Microwave Radiometer (DMR) instrument on the Cosmic Background Explorer (COBE). Due to the MAP project's limited mass, power, and financial resources, a traditional reliability concept including fully redundant components was not feasible. The MAP design employs selective hardware redundancy, along with backup software modes and algorithms, to improve the odds of mission success. In particular, MAP's propulsion system, which is used for orbit maneuvers and momentum management, uses eight thrusters positioned and oriented in such a way that its thruster-based attitude control modes can maintain three-axis attitude control in the event of the failure of any one thruster.

In March 2000, it was discovered that spacecraft configuration changes from the time of the attitude control system (ACS) critical design review had resulted in a migration of the spacecraft's center of mass $(C M)$ a significant distance in the spacecraft $Z$ axis. As a result of this CM migration, MAP no longer had a viable backup control mode in the event of a failure of the negative pitch axis thruster. It should be noted that at this time the propulsion system was fully integrated into the spacecraft. A team was put together to identify possible solutions to this problem; potential solutions identified included adding thruster plume shields to redirect thruster torque, adding or removing mass to the spacecraft, adding an additional thruster, moving thrusters, bending thrusters (either nozzles or propellant tubing), or accepting the loss of redundancy for this one thruster. The trade study looked at the impacts of each solution, including effects on the mass, cost, and fuel budgets, as well as schedule. It was decided to bend the thruster propellant tubing of the two roll control thrusters, which allowed that pair to be used for backup control in the negative pitch axis. After a further trade study by the ACS team, a bend angle of $10^{\circ}$ was selected.

Before any hardware was modified, Kennedy Space Center and Cape Canaveral Air Station Range Safety officials were consulted to get their approval on the post-bending test and verification plan. Once this plan was approved, a detailed procedure was written that included all aspects of the bending and testing effort. Since the propulsion subsystem was fully tested and integrated within the MAP spacecraft, the bending of the roll thrusters was performed in situ. The tubes that needed to be bent were in very close proximity to the spacecraft lower deck, tubing support brackets, and the thrusters themselves. The desired bend location was in the plane of the lower deck, with less than one inch of clearance between the tube and the deck edge. The extremely tight clearances and the requirement for a flight quality bend meant that standard tube bending equipment could not be used, so a custom bending tool was designed and fabricated. This bending tool was tested on flight tubing to determine its accuracy and effect on the strength 
of the bent tube. All sample bends were dye penetrant and burst tested with nominal results. None of the burst locations were in the bent region of the tubing.

The first phase of work on the spacecraft included removing multi-layered insulation (MLI), heaters, aluminum tape, wiring, etc., from the propellant lines which needed to be bent. The MLI was destroyed in the de-integration process. Heaters and other thermal circuits were removed or cut as required to gain access to the tubes that needed to be bent. The thrusters were unbolted from their original brackets, and the brackets were extracted. At this point, the thrusters were only attached to the MAP spacecraft via their feed tubes and electrical harnesses.

In the second phase, the bending tool was assembled in-place around the tube and thruster. Before the tube was bent, a measurement of the initial thruster orientation was taken to act as a starting reference. All orientation measurements were performed using optical theodolites which used a reference cube attached to the spacecraft and a flat mirror attached to the exit plane of the thruster nozzle. The bending tool was actuated by hand to execute the bend, which was measured frequently until the desired angle was reached.

After the tubes were bent, the bending tool was removed, and new thruster brackets were installed. Due to clearance issues, the new brackets incorporated a two-piece clamshell design to enable them to be installed around the bent thrusters. While the tubes were bare, a dye penetrant test on the tube surface was performed. A pressure test at maximum operating pressure ( 350 psig) was performed; a leak check was performed on the bent areas while the system was at maximum pressure. Finally, the heaters, wiring, thermal tape, and MLI were reintegrated and retested. All tests gave nominal results.

In addition to physically bending the thruster propellant tubing for two thrusters, it was necessary to perform an extensive redesign and reverification of the ACS thruster-based control modes. The thruster alignments used in the software had to be adjusted for the two bent thrusters, and the backup control logic for the negative pitch axis had to be changed to use a different thruster pair. In addition to these necessary changes, a number of other changes were implemented to provide a greater amount of flexibility when using the propulsion system. To provide a degree of control over the amount of pitch disturbance torque present when performing orbit maneuvers, a parameter was added to allow a fixed duty cycle to be set for any thruster pair. Also, the ability to specify differential gains for positive and negative torques in a given axis when using a backup control mode (these modes have less torque authority, in general) was added. Once the thruster mode algorithm changes were made and implemented in the MAP high fidelity (HiFi) simulation, it was a trivial matter to implement the changes in flight software, as the changes were made in the portion of the HiFi from which flight code was being automatically generated. An extensive series of tests were then conducted using the MAP flight software test facility (known as FlatSat), testing all changed and new capabilities to verify they performed as expected.

Photographs of the "before" and "after" thruster orientations will be shown, as well as information about the custom tool used to perform the bend. Extensive simulation and analytical results will be presented to demonstrate the effects of the CM migration, the efficacy of the thruster bend, and its impact on the spacecraft fuel budget. The flight performance verification plan that will be used to verify the expected performance of the reconfigured propulsion system 
and thruster control modes will be shown, along with results of early thruster operationsthruster one-shot firings, the initial momentum dump, thruster mode calibration burns--and orbit adjust and stationkeeping maneuvers.

Because of the importance and criticality of MAP's orbit adjust maneuvers (important because they were necessary to get the spacecraft to $L_{2}$ to conduct its science mission, and critical because the necessary $\Delta V$ could only be imparted to the spacecraft during its three phasing loops), it was very important to test the thruster modes and understand their performance onorbit. On the two days after launch, one-shot thruster checks were performed on each of the two attitude control electronics (ACE) boxes. At MAP's first apogee, the thruster configuration slated to be used for all of MAP's orbit maneuvers was used in a two-minute calibration burn. Figures 1 and 2 show the results of this calibration burn, comparing the predicted and actual thruster commands and attitude errors. As can be seen in these plots, the actual performance was significantly different than predicted, with a smaller duty cycle for $+Y$-axis thruster 4 and less pitch attitude error. This improved performance was welcome, but there was some concern that a reasonable explanation for it could be discovered. After analyzing the flight data and examining the relevant modeling parameters - primarily the expected spacecraft center of mass, thruster plume impingement torques, and relative thruster scale factors-a revised spacecraft model was derived. As shown in Figures 3 and 4, after these new model parameters were used, the HiFi results closely followed the actual flight data.

In the full paper, the flight data and resulting analysis from all three calibration burns and the orbit maneuvers will be examined and presented in detail, showing the exemplary performance of MAP's propulsion system and ACS thruster control modes. 


\title{
Restoring Redundancy to the MAP Propulsion System
}

\author{
James R. O'Donnell, Jr., Ph.D., Gary T. Davis, David K. Ward \\ NASA Goddard Space Flight Center \\ Greenbelt, MD 20771 USA
}

\section{OUTLINE}

1. MAP Mission Overview

1.1. Science Objectives

1.2. Nominal Propulsion/ACS Design

2. CM Migration Problem

2.1. Pitch Axis Stability Problem in Backup Mode

2.2. Solution Trade Study

2.2.1. Plume Deflector Shields

2.2.2. Add or Remove Mass

2.2.3. Sacrifice Pitch Axis Backup Mode

2.2.4. Bend Thruster Nozzles

2.2.5. Move Thrusters 5/6/7/8

2.2.6. Bend Thrusters $1 / 2$

3. Bending Operation

3.1. De-Integration

3.2. Bending

3.2.1. Bend Tool Design

3.2.2. Bend Tool Operation and Performance

3.3. Post-Bend Verification

3.3.1. Bend Angle

3.3.2. Pressure Test

3.3.3. Leak Test

3.4. Re-Integration

4. ACS Redesign

4.1. Bend Angle Trade Study

4.1.1. CM Uncertainty

4.1.2. Plume Impingement

4.1.3. $5^{\circ}$ vs $10^{\circ}$ Bend

4.2. Thruster Mode Redesign

4.2.1. Thruster Bend Software Implementation

4.2.2. Delta V Duty Cycle

4.2.3. Backup Control Gains

4.3. Thruster Mode Testing and Verification

4.3.1. HiFi Tests

4.3.2. FlatSat Flight Software Regression Testing

5. Flight Performance Verification Plan

6. Flight Tests and Calibration

7. MAP Orbit Adjust and Stationkeeping Maneuvers

8. Conclusion 


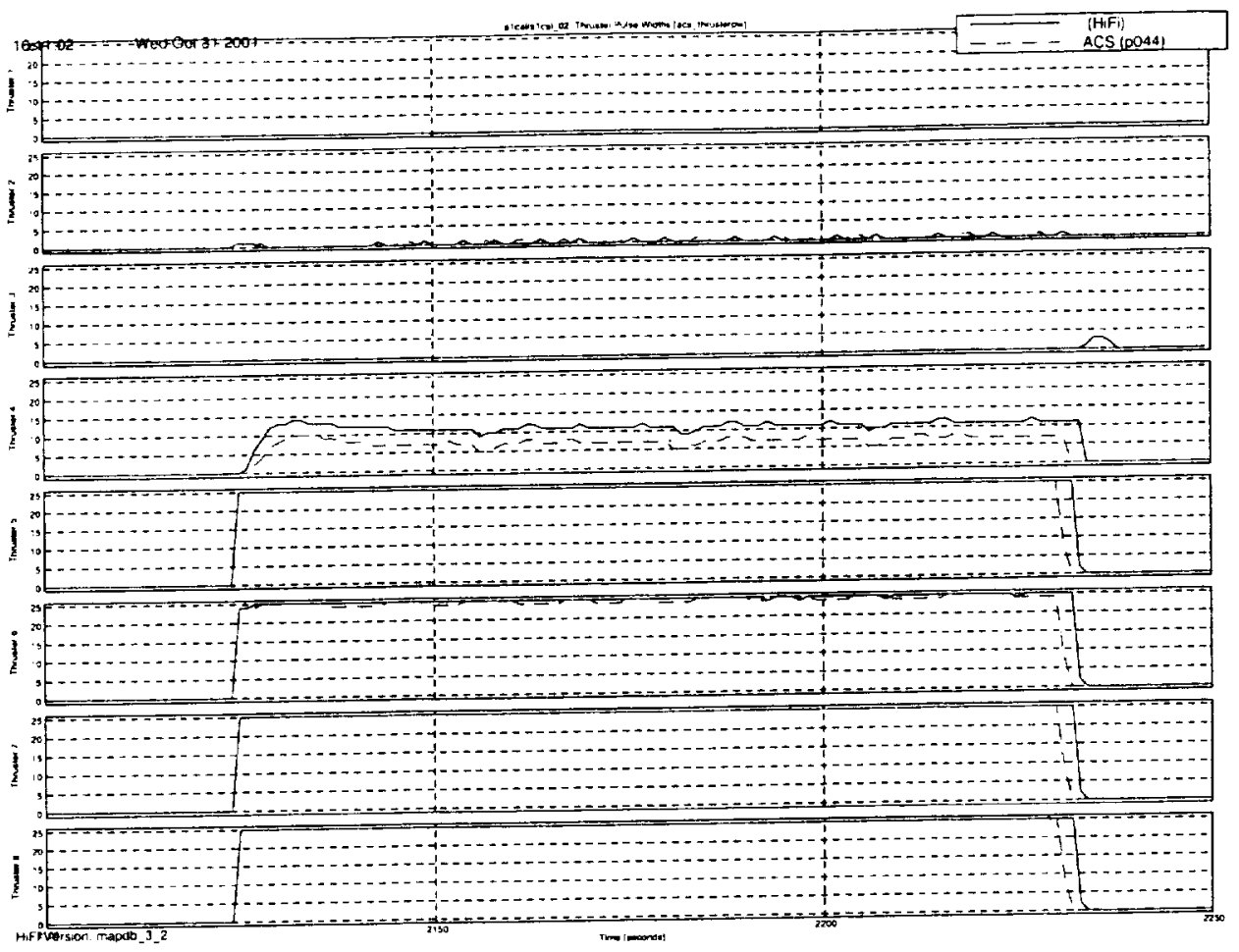

Figure 1: A1 Calibration Burn, Predicted vs Actual Thruster Commands (Pre-Calibration)

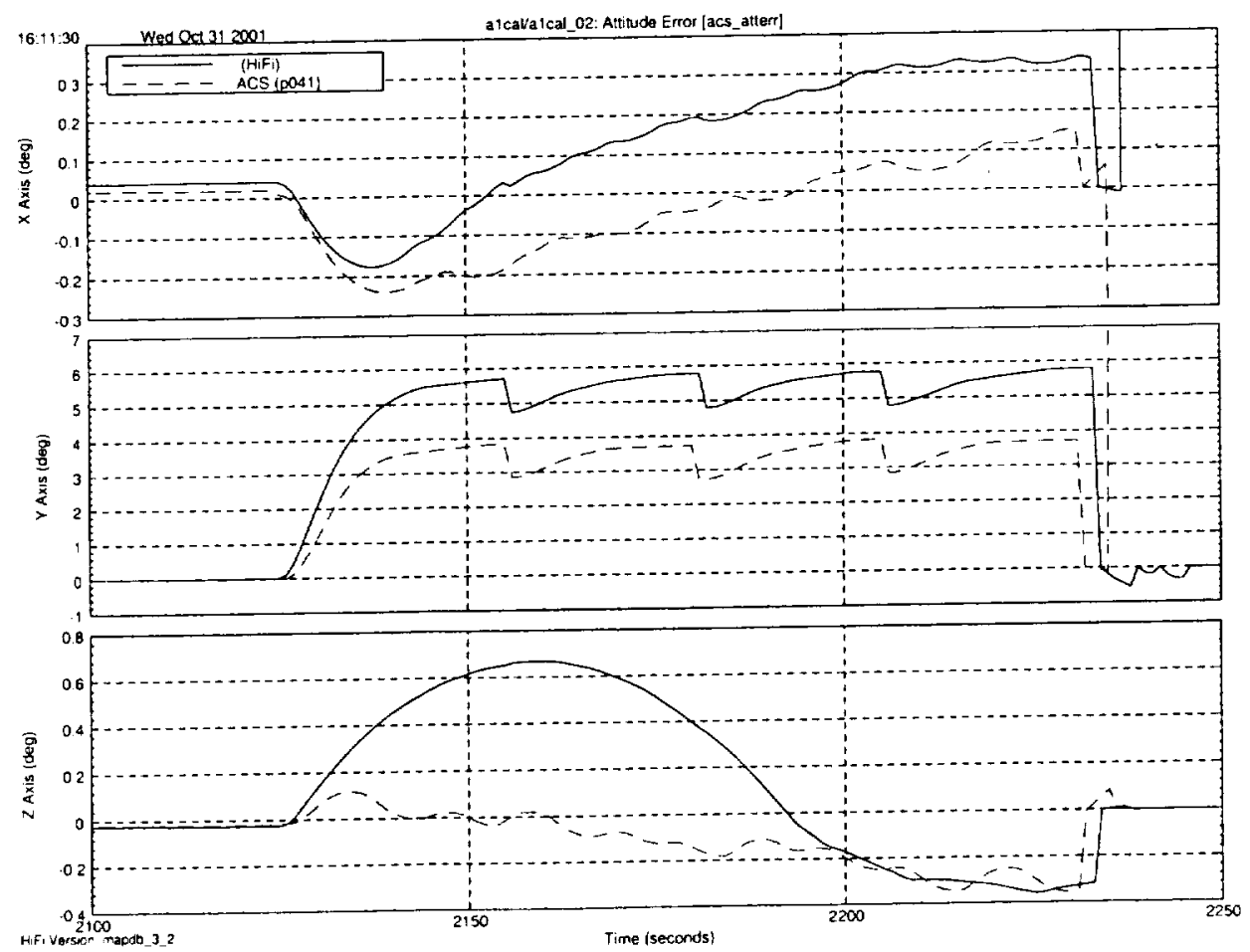

Figure 2: A1 Calibration Burn, Predicted vs Actual Attitude Errors (Pre-Calibration) 


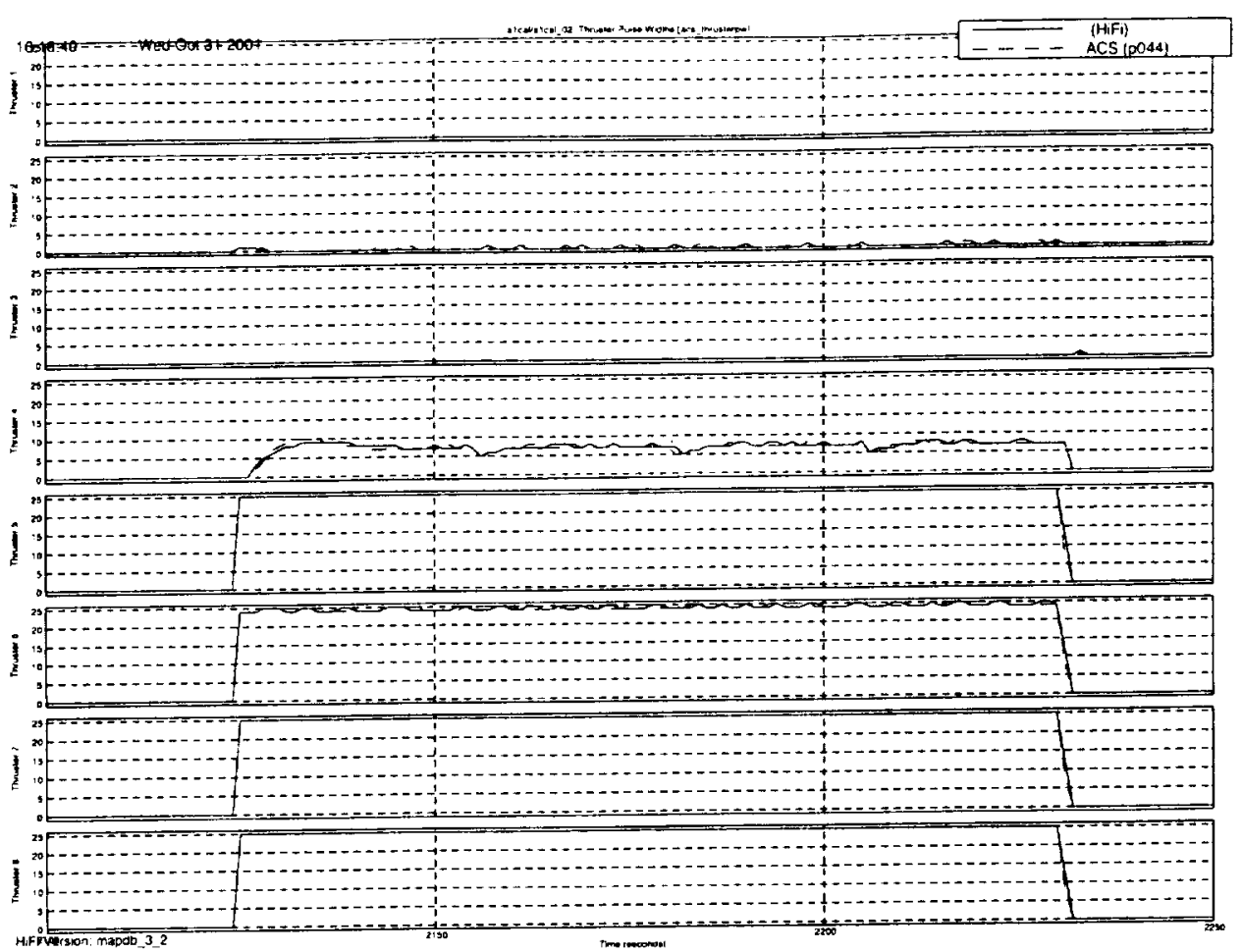

Figure 3: A1 Calibration Burn, Predicted vs Actual Thruster Commands (Post-Calibration)

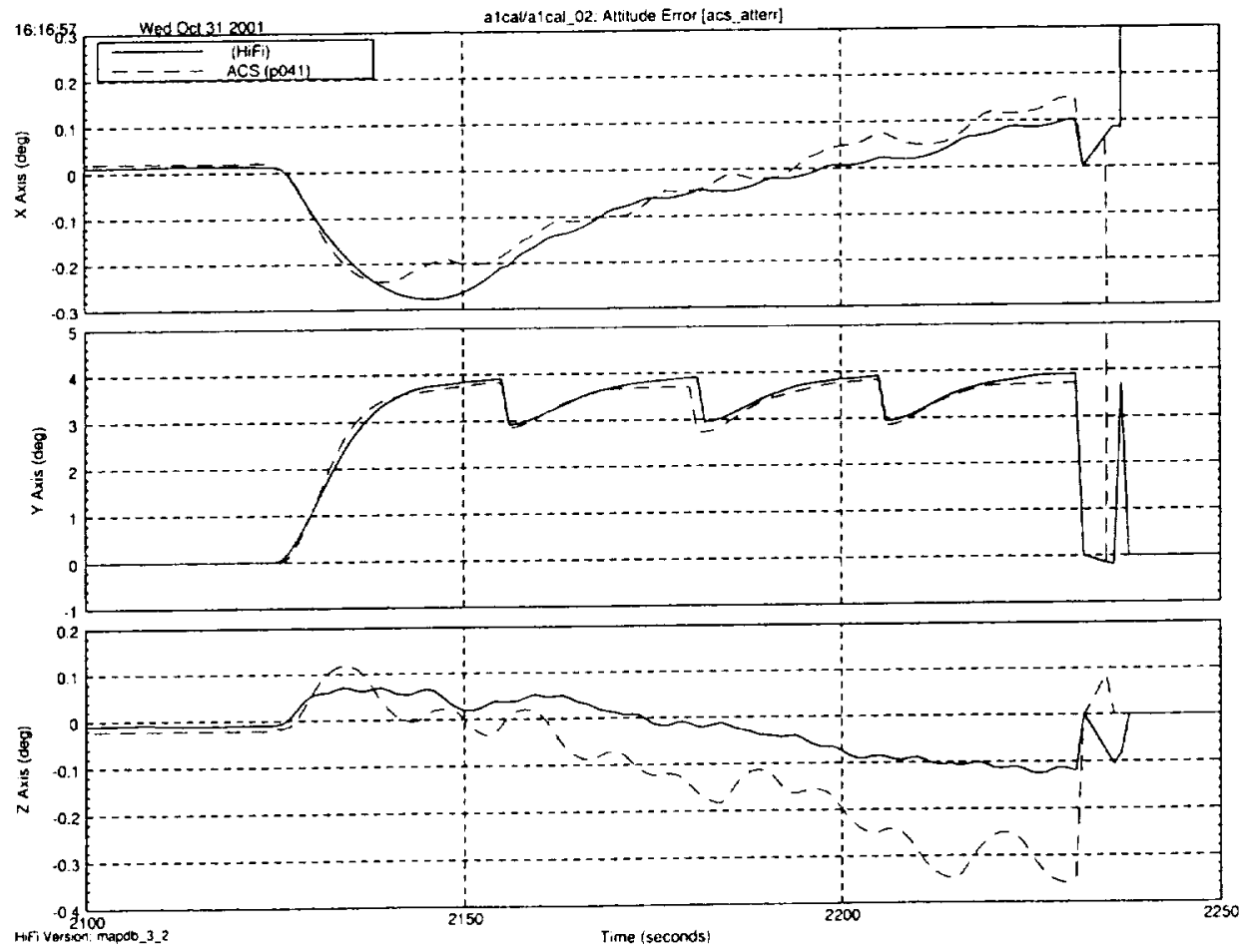

Figure 4: A1 Calibration Burn, Predicted vs Actual Attitude Errors (Post-Calibration) 